\section{KOMPASS}

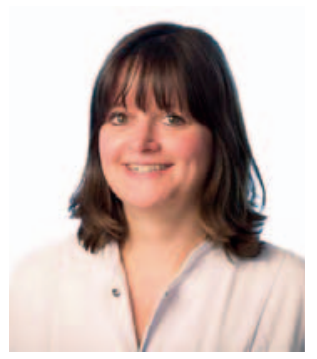

\section{Petra Voiß}

Integrative Onkologie/Naturheilkunde, Klinik für Naturheilkunde und Integrative Medizin, Kliniken Essen-Mitte, Evang. Huyssens Stiftung/Knappschaft GmbH, Essen, Deutschland

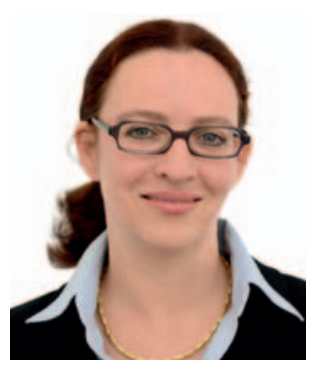

\section{Claudia M. Witt}

Institut für komplementäre und integrative Medizin, UniversitätsSpital Zürich und Universität Zürich, Zürich, Schweiz

\title{
Das Potential von Mind Body Medicine in der Onkologie
}

\section{Warum Integrative Onkologie?}

«Was kann ich selber tun, um meinen Krankheitsverlauf positiv zu beeinflussen?» fragen Patienten nicht selten ihre behandelnden Onkologen und hoffen auf Rat. Umfragen zufolge nutzt ca. die Hälfte der Krebspatienten Komplementärmedizin [1] und drei Viertel der Patientinnen mit fortgeschrittenem Mammakarzinom interessieren sich für Komplementärmedizin [2]. Der Sammelbegriff «Komplementärmedizin» schließt eine Vielzahl unterschiedlicher Verfahren ein, die üblicherweise nicht zur Schulmedizin gerechnet [3] und zusätzlich zur antitumoralen Therapie eingesetzt werden. Daten aus dem amerikanischen nationalen Krebsregister zeigten, dass eine Anwendung anstelle von antitumoraler Therapie mit höherer Sterblichkeit einherging (Hazard Ratio 2,50 [4]) und dass auch die ergänzende Nutzung von «nicht-evidenzbasierte Krebstherapien, die durch nichtmedizinisches Personal verabreicht wurden» negative Folgen haben kann. Wegen einer schlechteren Adhärenz bei den onkologischen Therapien war die 5-Jahres-Überlebensrate 4\% niedriger [5].

Zwar nutzten nur 0,01\% der Patienten diese nicht-evidenzbasierten Therapien, die negativen Folgen unterstreichen jedoch, wie wichtig eine Integration des Themas Komplementärmedizin in die onkologische Therapie ist. Zudem wurde in einer deutschlandweiten Umfrage mit 700 Brustkrebspatientinnen am häufigsten der Wunsch nach einer «integrativen» Behandlung geäußert [6]. Dem trägt das aus den USA stammende Konzept einer Integrativen Onkologie (Box 1) Rechnung, die sich darum be-
Integrative Onkologie ist ein patientenzentriertes, evidenzinformiertes Gebiet der Krebstherapie, das Mind-Body-Verfahren, natürliche Produkte und/oder Lebensstil-Änderungen aus unterschiedlichen Traditionen begleitend $z u$ den konventionellen Krebstherapien einsetzt. Die Integrative Onkologie versucht, Gesundheit, Lebensqualität und klinische Outcomes über den Behandlungsverlauf hinweg zu optimieren und Menschen zu befähigen, Krebs vorzubeugen und zu aktiven Teilnehmern vor und während der Krebsbehandlung, sowie über diese hinaus zu werden.

Box 1. Definition Integrative Onkologie der Society for Integrative Oncology [23].

müht, die antitumorale Therapie sinnvoll mit Komplementärmedizin zu ergänzen.

\section{Mind Body Medicine}

Das therapeutische Vorgehen im Rahmen der Mind Body Medicine (MBM) und die dafür eingesetzten Verfahren ermöglichen ein patientenzentriertes, evidenzbasiertes Vorgehen. Angesichts der aktuellen Datenlage sind Empfehlungen zu einem gesundheitsfördernden Lebensstil wichtiger Bestandteil einer onkologischen Beratung. In der MBM werden zudem die oftmals separierten Bereiche Bewegung, Ernährung und Stressreduktion zusammengeführt und dem $\mathrm{Pa}$ tienten als umfassende Selbsthilfestrategie vermittelt. Der Fokus liegt dabei auf einer Stärkung der Gesundheitsressourcen über die Grunderkrankung hinaus. Die Patienten werden unterstützt, das eigene Verhalten und das Lebensumfeld unter Berücksichtigung der individuellen Möglichkeiten und Bedürfnisse so gesundheitsfördernd wie möglich zu gestalten und mehr Selbstwirk-

\section{KARGER}

Fax +497614520714 information@karger.com www.karger.com (c) 2018 S. Karger GmbH, Freiburg
Prof. Dr. Claudia M. Witt

Institut für komplementäre und integrative Medizin

UniversitätsSpital Zürich

Rämistraße 100, 8091 Zürich, Schweiz

claudia.witt@uzh.ch 
samkeit zu entwickeln. MBM ist kein starres Konzept, sondern orientiert sich an den drei Säulen der evidenzbasierten Medizin: Werte und Wünsche der Patienten, ärztliche Expertise und wissenschaftliche Erkenntnisse. Das Therapiespektrum ist nicht-medikamentös ausgerichtet und reicht von gesundheitsfördernder Lebensstilmodifikation durch Bewegung (inkl. Yoga, Qigong, Tai-Chi), Ernährung und Entspannung (z.B. Progressive Muskelentspannung, Imaginationsverfahren) bis hin zu «Mindfulness Based Stress Reduction» (MBSR)-Programmen und dem Erlernen von evidenzbasierten komplementärmedizinischen Selbsthilfemaßnahmen wie z.B. Akupressur bei tumorassoziierter Fatigue [7].

\section{Integration in die Klinik}

Integration von MBM in die Versorgung kann in unterschiedlichen Formen stattfinden. Am interdisziplinären Brustzentrum der Kliniken Essen Mitte in Deutschland wird unter Leitung von Professor Kümmel das «Beste aus beiden Welten» direkt im Brustzentrum kombiniert. Allen Patientinnen wird eine entsprechende ambulante Beratung nach der Tumorkonferenz angeboten. Alle stationären Patientinnen werden zudem von Ärzten aus der Abteilung von Professor Dobos für Naturheilkunde und Integrative Medizin visitiert und gegebenenfalls behandelt [8]. Begleitend zur Chemotherapie besteht die Möglichkeit an einem MBM-Gruppenprogramm teilzunehmen und zur Minderung von therapieassoziierten Nebenwirkungen auch andere evidenzbasierte komplementärmedizinische Verfahren, wie z.B. Akupunktur, zu erhalten. MBM-Programme werden auch für Patientinnen unter endokriner Therapie und in metastasierter Situation angeboten. In Deutschland wird an der Universität Duisburg-Essen seit 13 Jahren eine Fortbildung in MBM angeboten [9].

Am Universitätsspital Zürich können die Patienten dem Institut für komplementäre und integrative Medizin zugewiesen werden, das eng mit dem Comprehensive Cancer Center Zürich und den onkologischen Kliniken zusammenarbeitet. Die MBM wird sowohl im Gruppen- als auch Einzelsetting angeboten und wenn sinnvoll, mit anderen evidenzbasierten komplementärmedizinischen Verfahren, wie z.B. Hypnose oder Akupunktur, kombiniert. Im Zusammenarbeit mit dem Schweizer Fachverband Mind Body Medicine wird seit 2018 an der Universität Zürich eine Weiterbildung als Certificate of Advanced Studies in Gesundheitspsychologischer Lebensstiländerung und Mind Body Medicine [10] angeboten.

\section{Barrieren der Integration beseitigen}

Seitens der Patienten besteht der Wunsch nach Integration und es gibt Verfahren die sicher sind und sich in klinischen Studien wirksam gezeigt haben. Deshalb stellt sich die Frage, weshalb die gewünschte Integration in die onkologische Versorgung so wenig stattgefunden hat.

Die Onkologie gehört zu den medizinischen Bereichen mit dem schnellsten Wissenszuwachs und stellt damit bereits hohe Anforderungen an die Ärzte, um up to date zu bleiben. Wenn sie komplementärmedizinische Beratungen durchführen müssen, ohne
Tabelle 1. Empfehlungen zu MBM Verfahren bei Brustkrebs

\begin{tabular}{lll}
\hline Indikation & Therapie & $\begin{array}{l}\text { Empfehlungs- } \\
\text { grad [19] }\end{array}$ \\
\hline Angst, Stress & Meditation / MBSR & A \\
& Yoga & B \\
& Stressreduktion & B \\
& Entspannungsverfahren & C \\
Fatigue & Yoga & C \\
Schlafstörungen & Qigong /Tai-Chi & C \\
Depression, Stimmmungs & Yoga & Meditation (insb. MBSR) \\
schwankungen & A \\
& Yogtspannungsverfahren & A \\
& Stressmanagement & B \\
Übelkeit, Erbrechen & Akupressur & B \\
Lebensqualität & Meditation / MBSR & A \\
& Yoga & B \\
& Qigong & C \\
& Stressmanagement & C
\end{tabular}

Empfehlungsgrade: A: wird empfohlen und sollte angeboten werden (große Sicherheit, dass der Nutzen substantiell ist), B: wird empfohlen und sollte angeboten werden (große Sicherheit, dass der Nutzen moderat ist oder moderate Sicherheit, dass der Nutzen moderat bis substantiell ist), C: ausgewählten Patienten basierend auf professionellen Entscheidungen und Patientenpräferenzen anbieten (mindestens moderate Sicherheit, dass ein kleiner Nutzen vorhanden ist).

dass sie die entsprechende Expertise haben, ist dies nicht nur ein Qualitätsproblem, sondern auch ein zusätzlicher Stressor für die behandelnden Ärzte. Das Förderprojekt «Kompetenznetzwerk Komplementärmedizin in Onkologie» [11] der Deutschen Krebshilfe leistet aktuell einen Beitrag, um diese Situation zu verbessern, indem es auch Trainings für in der Onkologie tätige Berufsgruppen entwickelt, evaluiert und implementiert.

\section{Evidenz der Mind Body Medicine}

Bezüglich der evidenzbasierten Empfehlungen zu Ernährung und Bewegung möchten wir auf die gültigen Leitlinie [12] und die Empfehlungen des Word Cancer Research Funds [13] verweisen. Es gilt mittlerweile als gesichert, dass Stress, Ängstlichkeit und Depressivität sich negativ auf den Krankheitsverlauf auswirken [14-16] und es gibt Hinweise, dass sich eine hohe Stressbelastung während der ersten Monate nach Diagnosestellung ungünstig auf die zukünftige Lebensqualität, z.B. Schmerzen und Fatigue, auswirkt [17]. Insbesondere für diese Symptomatiken haben sich Verfahren der MBM wirksam gezeigt. Die American Society for Clinical Oncology hat die Empfehlungen der Society for Integrative Oncology (SIO) $[18,19]$ befürwortet. Demnach sollte allen Brustkrebspatientinnen folgendes empfohlen werden (Empfehlungsgrad A) :

- Meditation und MBSR zur Verbesserung der Lebensqualität.

- Meditation und MBSR zur Verbesserung von Stimmungsschwankungen, depressiven Symptomen und Angst sowie zur Stressbewältigung.

- Entspannungstraining zur Verbesserung von Stimmungsschwankungen und depressiven Symptomen. 
Auch für andere Verfahren der MBM liegen positive Ergebnisse aus klinischen Studien vor (Tab. 1). Auch wenn viele der Studien mit Brustkrebspatientinnen durchgeführt wurden, gibt es auch Daten zu anderen Tumorentitäten, wie die Artikel in dieser Ausgabe zeigen:

Die Übersichtsarbeit von Duncan et al. [20] gibt einen guten Überblick über die Evidenz zu nicht-pharmakologischen Interventionen zur Verbesserung der Lebensqualität von Cancer-Survivors.

In der Rubrik Erfahrung aus der Praxis [21] wird das Konzept des Stressbewältigungsprogramms für onkologische Patienten der Kliniken Essen-Mitte dargestellt. Und schließlich wird der Einfluss von Yogatherapie auf Angst bei onkologischen Patienten auf Grundlage der randomisiert kontrollierten Studie von Hardoerfer et al. [22] diskutiert.
Wir hoffen, dass Ihnen diese Ausgabe neue Impulse für eine integrative Behandlung Ihrer Patienten gibt!

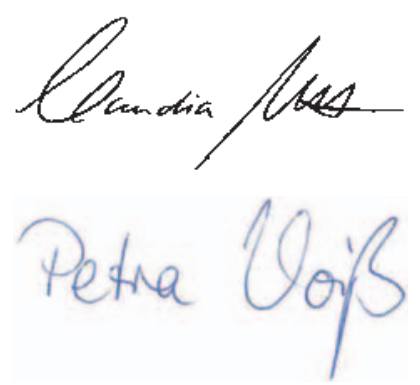

Claudia M. Witt und Petra Voiß

\section{Literatur}

- 1 Horneber M, Bueschel G, Dennert G, et al. How many cancer patients use complementary and alternative medicine: a systematic review and metaanalysis. Integr Cancer Ther 2012;11: 187-203.

2 Fremd C, Hack CC, Schneeweiss A, et al.: Use of complementary and integrative medicine among German breast cancer patients: predictors and implications for patient care within the PRAEGNANT study network. Arch Gynecol Obstet 2017;295:1239-1245.

3 Willich SN: Schulmedizin und Komplementärmedizin - Verständnis und Zusammenarbeit müssen vertieft werden: Schlusswort. Dtsch Arztebl Int 2004;101:2315.

4 Johnson SB, Park HS, Gross CP, et al.: Use of alternative medicine for cancer and its impact on survival. J Natl Cancer Inst 2018;110:121-124.

5 Johnson SB, Park HS, Gross CP, et al.: Complementary medicine, refusal of conventional cancer therapy, and survival among patients with curable cancers. JAMA Oncol 2018; doi:10.1001/jamaoncol.2018.2487.

-6 Oskay-Ozcelik G, Lehmacher W, Konsgen D, et al.: Breast cancer patients' expectations in respect of the physician-patient relationship and treatment management results of a survey of 617 patients. Ann Oncol 2007;18:479-484.

7 Zick SM, Sen A, Wyatt GK, et al.: Investigation of 2 types of self-administered acupressure for persistent cancer-related fatigue in breast cancer survivors: a randomized clinical trial. JAMA Oncol 2016;2:1470-1476.

$>8$ Dobos GJ, Voiss P, Schwidde I, et al.: Integrative oncology for breast cancer patients: introduction of an expert-based model. BMC Cancer 2012;12:539.
9 Vertiefungsseminar der Mind-Body-Medizin, 2018. www.nhk-fortbildungen.de/18-0-MindBody-Medicine-Vertiefungsseminar-AktiveFortbildung-fuer-Mediziner-und-Therapeuten. html (Zugriff: 20.08.2018).

10 CAS in Gesundheitspsychologischer Lebensstiländerung und Mind Body Medicine, 2018. https://www.psychologie.uzh.ch/de/bereiche/sob/ angsoz/CAS/2018.html (Zugriff: 20.08.2018).

11 Kompetenznetz Komplementärmedizin in der Onkologie, 2018. www.kompetenznetz-kokon. de (Zugriff: 20.08.2018).

12 Arends J, Bertz H, Bischoff SC, et al.: S3-Leitline der Deutschen Gesellschaft für Ernährungsmedizin e.V. (DGEM) in Kooperation mit der Deutschen Gesellschaft für Hämatologie und Onkologie e.V. (DGHO), der Arbeitsgemeinschaft 'Supportive Maßnahmen in der Onkologie, Rehabilitation und Sozialmedizin' der Deutschen Krebsgesellschaft (ASORS) und der Österreichischen Arbeitsgemeinschaft für klinische Ernährung (AKE). Aktuell Ernährungsmed 2015;40:e1-e74.

13 World Cancer Research Fund /American Institute for Cancer Research. Diet, Nutrition, Physical Activity and Cancer: a Global Perspective. Continuous Update Project Expert Report 2018. www.dietandcancerreport.org. (Zugriff: 20.08.2018).

14 Chida Y, Hamer M, Wardle J, et al.: Do stressrelated psychosocial factors contribute to cancer incidence and survival? Nat Rev Clin Oncol 2008;5:466-475.

15 Pinquart M, Duberstein PR: Depression and cancer mortality: a meta-analysis. Psychol Med 2010;40:1797-1810.
16 Satin JR, Linden W, Phillips MJ: Depression as a predictor of disease progression and mortality in cancer patients: a meta-analysis. Cancer 2009;115:5349-5361.

17 Harris LN, Bauer MR, Wiley JF, et al.: Chronic and episodic stress predict physical symptom bother following breast cancer diagnosis. J Behav Med 2017;40:875-885.

18 Lyman GH, Bohlke K, Cohen L: Integrative therapies during and after breast cancer treatment: ASCO endorsement of the SIO clinical practice guideline summary. J Oncol Pract 2018;14:495-499.

19 Greenlee H, DuPont-Reyes MJ, Balneaves LG, et al.: Clinical practice guidelines on the evidence-based use of integrative therapies during and after breast cancer treatment. CA Cancer J Clin 2017;67:194-232.

20 Duncan M, Moschopoulou E, Herrington E, et al.: Review of systematic reviews of non-pharmacological interventions to improve quality of life in cancer survivors. BMJ Open 2017; 7:e015860.

21 Paul A, Cramer H, Lauche R, et al.: An oncology mind-body medicine day care clinic: concept and case presentation. Integr Cancer Ther 2013;12:503-507.

22 Hardoerfer K, Jentschke E: Effect of yoga therapy on symptoms of anxiety in cancer patients. Oncol Res Treat 2018;41:DOI: 10.1159/000488989.

23 Witt CM, Balneaves LG, Cardoso MJ, et al.: A Comprehensive definition for integrative oncology. J Natl Cancer Inst Monogr 2017;2017: $\operatorname{lgx} 012$. 